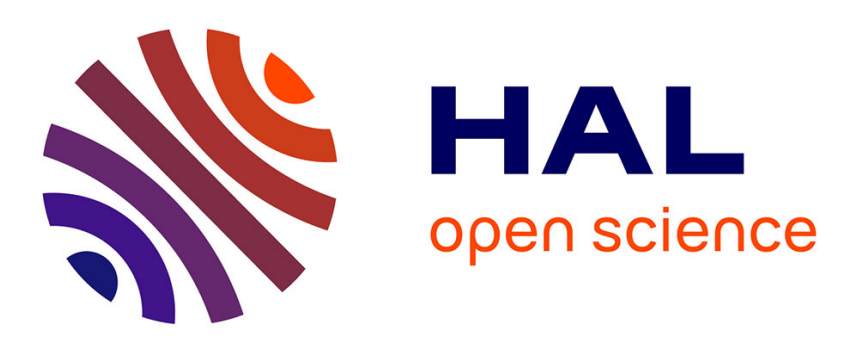

\title{
Structure of the Magnetic Reconnection Diffusion Region from Four-Spacecraft Observations
}

\author{
A. Vaivads, Y. Khotyaintsev, M. André, A. Retinò, S. Buchert, B. Rogers, \\ Pierrette Décréau, G. Paschmann, T. Phan
}

\section{To cite this version:}

A. Vaivads, Y. Khotyaintsev, M. André, A. Retinò, S. Buchert, et al.. Structure of the Magnetic Reconnection Diffusion Region from Four-Spacecraft Observations. Physical Review Letters, 2004, 93 (10), 10.1103/PhysRevLett.93.105001 . insu-02873931

\section{HAL Id: insu-02873931 https://hal-insu.archives-ouvertes.fr/insu-02873931}

Submitted on 8 Jul 2020

HAL is a multi-disciplinary open access archive for the deposit and dissemination of scientific research documents, whether they are published or not. The documents may come from teaching and research institutions in France or abroad, or from public or private research centers.
L'archive ouverte pluridisciplinaire $\mathbf{H A L}$, est destinée au dépôt et à la diffusion de documents scientifiques de niveau recherche, publiés ou non, émanant des établissements d'enseignement et de recherche français ou étrangers, des laboratoires publics ou privés. 


\title{
Structure of the Magnetic Reconnection Diffusion Region from Four-Spacecraft Observations
}

\author{
A. Vaivads, Y. Khotyaintsev, M. André, A. Retinò, and S. C. Buchert \\ Swedish Institute of Space Physics, Uppsala, Sweden \\ B. N. Rogers \\ Department of Physics and Astronomy, Dartmouth College, Hanover, New Hampshire, USA \\ P. Décréau \\ LPCE/CNRS and University of Orléans, Orléans, France
}

G. Paschmann

Max Planck Institute for Extraterrestrial Physics, Garching, Germany

T. D. Phan

Space Sciences Laboratory, Berkeley, California, USA

(Received 22 April 2004; published 31 August 2004)

\begin{abstract}
Magnetic reconnection leads to energy conversion in large volumes in space but is initiated in small diffusion regions. Because of the small sizes of the diffusion regions, their crossings by spacecraft are rare. We report four-spacecraft observations of a diffusion region encounter at the Earth's magnetopause that allow us to reliably distinguish spatial from temporal features. We find that the diffusion region is stable on ion time and length scales in agreement with numerical simulations. The electric field normal to the current sheet is balanced by the Hall term in the generalized Ohm's law, $E_{n} \sim \mathbf{j} \times \mathbf{B} / n e \cdot \hat{\mathbf{n}}$, thus establishing that Hall physics is dominating inside the diffusion region. The reconnection rate is fast, $\sim 0.1$. We show that strong parallel currents flow along the separatrices; they are correlated with observations of high-frequency Langmuir/upper hybrid waves.
\end{abstract}

DOI: 10.1103/PhysRevLett.93.105001

PACS numbers: 94.30.Di, 52.35.Hr, 52.35.Vd

Magnetic reconnection in astrophysical plasma environments allows fast conversion of the magnetic field energy of two colliding magnetized plasmas into kinetic energy of ions and electrons. Energy and plasma from the solar wind can enter the Earth's magnetosphere mainly due to magnetic reconnection between the magnetic fields of the solar wind and the Earth's magnetosphere at the magnetopause [1,2]. The reconnection is initiated in small diffusion regions, where the magnetic flux is no longer frozen into the motion of the ions (ion diffusion region) and the electrons (electron diffusion region). The ion diffusion region (we refer to it simply as the diffusion region) is larger than the electron diffusion region. Single spacecraft observations have established the collisionless character of the diffusion region, particularly based on observations of an out-of plane component of the magnetic field [2-4]. However, the single satellite measurements involve ambiguity in distinguishing spatial and temporal features of the diffusion region [2]. For a detailed description, and reliable comparison with numerical simulations and theories, it is important to use high quality multipoint observations of the diffusion region.

On 20 February 2002 around 13-14 UT the four Cluster spacecraft [5] crossed the magnetopause many times tailward and duskward of the cusp (Fig. 1). We investigate one of the magnetopause crossings at
13:22 UT when the Cluster spacecraft cross the diffusion region. The separation between the spacecraft is about $100 \mathrm{~km}$ which is comparable to the ion inertial length $\lambda_{i} \sim 75 \mathrm{~km}$. We focus on features on scales in between the ion and electron scales, or larger.

The left side of Fig. 2 shows results from a 2D two fluid MHD numerical simulation of the diffusion region near the $X$ line [6], plasma parameters are similar to those in our observations. The color coding (shading) shows the out-of-plane magnetic field component (often referred to as the Hall magnetic field). The quadrupolar structure is characteristic for symmetric collisionless reconnection $[4,7]$. Symmetric means that the density and magnitude of the magnetic field is the same on both sides of the current sheet. The inset shows the Cluster configuration and schematic trajectory through the diffusion region. Panel 2(a) shows the reconnecting magnetic field components $B_{L}$ as observed by the four Cluster spacecraft. The time delay between the current sheet crossings gives the normal and velocity of the magnetopause, $V_{m p} \approx-120$ $\left(\begin{array}{ll}0.82 & 0.54-0.18\end{array}\right) \mathrm{km} / \mathrm{s}$ GSE. Knowing this velocity reliably from four-spacecraft measurements we can establish the spatial scale of the magnetopause current layer, shown at the bottom of Fig. 2. The current sheet thickness is about $300 \mathrm{~km}, \sim 4 \lambda_{i}$. All four-spacecraft observe a very similar structure of the current sheet 


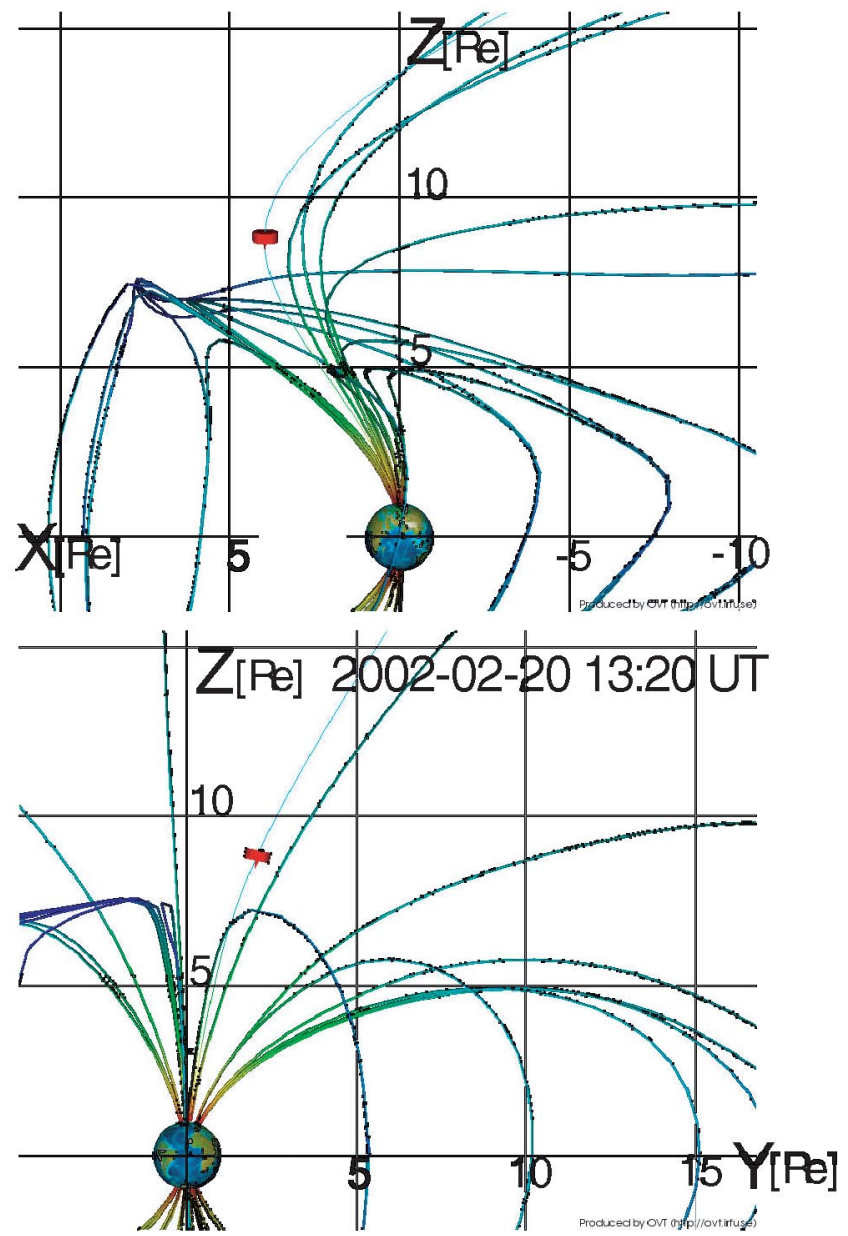

FIG. 1 (color online). Location of the four Cluster spacecraft (red circle) and the magnetic field lines of the Earth's magnetosphere from the Tsyganenko 2001 model (interplanetary magnetic field IMF in GSE $\left(B_{X}, B_{Y}, B_{Z}\right)=(0,7,-2) \mathrm{nT}$, and solar wind pressure $3.5 \mathrm{nPa}$ are observed averages from the ACE spacecraft). Assuming that reconnection occurs in regions with antiparallel magnetic fields, the varying IMF $B_{Z}$ (from +3 to $-7 \mathrm{nT}$ within $10 \mathrm{~min}$ ) is consistent with a diffusion region occurring duskward and tailward of the cusp, as observed by Cluster.

[panel 2(a)]; the largest deviation is seen by C3. Thus, the current sheet has a planar structure on the scale of the spacecraft separation $\left(\sim \lambda_{i}\right)$ and is stable on the time scale of $1 \mathrm{~s}$ (approximately the ion gyroperiod). The current sheet is bifurcated, with the current (gradient in $B_{L}$ ) being strongest along the outer edges. Such determination of the spatial structure of the diffusion region is only possible with multispacecraft measurements.

The out-of-plane magnetic field component $B_{M}$ [panel 2(b)] shows a bipolar variation with the highest amplitude being $\sim 50 \%$ of $B_{L}$ outside the current sheet. According to numerical simulations, the bipolar variation in $B_{M}$ is an indication of the ion diffusion region in collisionless reconnection and has been used as one of the arguments for ongoing reconnection in previous studies $[3,8]$. The fact that all four-spacecraft, crossing the magnetopause consecutively, observed Hall fields of this large amplitude indicates that this is a stable spatial feature of the diffusion region rather than some brief temporal variation. There is no significant constant offset in $B_{M}$, a so-called guide field. The presence of a nonzero normal component of the magnetic field $B_{N} \sim-3 \mathrm{nT}$ [panel 2(c)] inside the current sheet also suggests ongoing reconnection. Inside the current sheet $B_{N}$ is a major fraction of the total magnetic field and the observed magnitudes agree with the simulation, while good agreement can not be expected when $B_{N}$ is a small fraction of the total field. The negative sign of $B_{N}$, and the fact that a negative $B_{M}$ is followed by a positive, are both consistent with Cluster crossing the diffusion region south of the $X$ line.
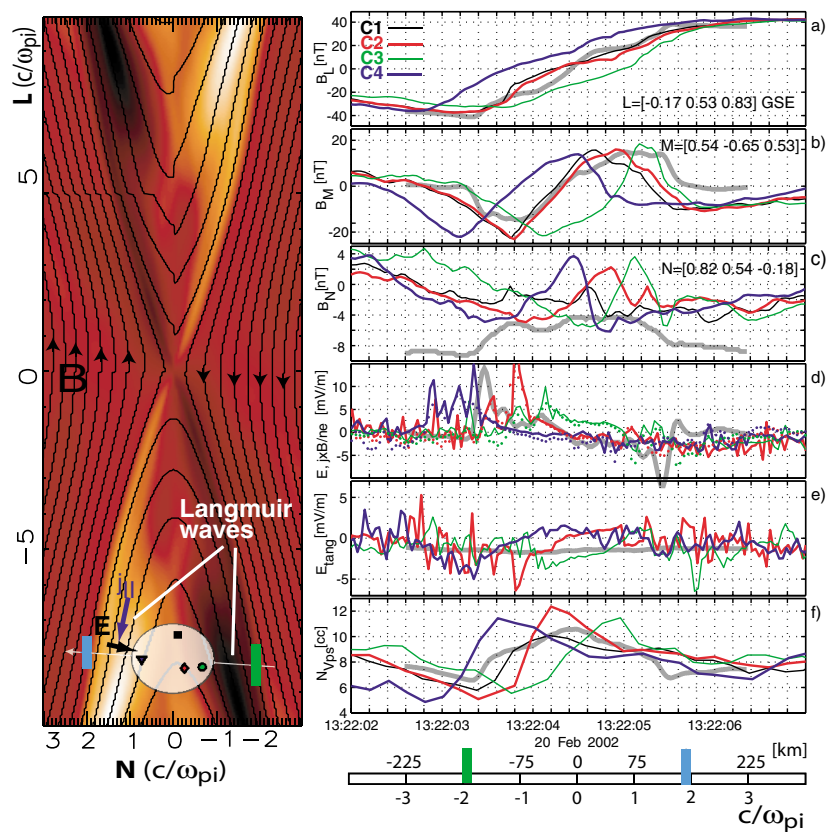

FIG. 2 (color online). Left: The structure of the diffusion region from a numerical simulation. The magnetic field lines are shown, and the out-of-plane magnetic field is color coded (white is positive and black is negative). Also shown is the projection of the Cluster configuration and the approximate location relative to the diffusion region $(\mathrm{C} 1$ : black square; $\mathrm{C} 2$ : red diamond; C3: green circle; C4: blue triangle). Right: Cluster observations; simulation results are shown with gray thick lines. (a) Reconnecting magnetic field component. (b) Out-of-plane magnetic field component. (c) Normal magnetic field component. (d) Electric field normal to the magnetopause, directly observed $E_{n}$ (solid lines), $\mathbf{j} \times \mathbf{B} / n e \cdot \hat{\mathbf{n}}$ (dotted lines). (e) Tangential electric field; the average value is about $-1 \mathrm{mV} / \mathrm{m}$. (f) plasma density from the satellite potential. At the bottom the spatial scale obtained from the four-spacecraft magnetopause velocity estimate is given. The observations are consistent with fast collisionless reconnection. 
The solid lines in panel 2(d) show the electric field component normal to the magnetopause, $E_{n}$ (the electric field is measured in the satellite spin plane and $E_{n}$ is along the direction in the spin plane closest to the magnetopause normal, here 5 degrees off from the nominal normal). $E_{n}$ changes sign from positive to negative in the center of the current sheet. The dotted lines show $\mathbf{j} \times$ $\mathbf{B} / n e \cdot \hat{\mathbf{n}}$, indicating how much of $E_{n}$ is balanced by the Hall term in the generalized Ohm's law [9]. There is good agreement between $E_{n}$ and $\mathbf{j} \times \mathbf{B} / n e \cdot \hat{\mathbf{n}}$ within the narrow region of strong $E_{n}$ at 13:22:04 UT. This observationally confirms the major role of the Hall term in the formation of the structure of the diffusion region. The cross-field potential drop across the region of strong electric field is $\sim 300 \mathrm{~V}$. A proton accelerating through $300 \mathrm{~V}$ would obtain a speed of $\sim 250 \mathrm{~km} / \mathrm{s}$ which is approximately the Alfvén velocity in the inflow region and comparable to the predicted outflow velocity of ions from the reconnection region.

If the magnetopause locally is a rotational discontinuity, then $B_{N}$ being $\sim 10 \%$ of $B_{L}$ gives a reconnection rate of $\sim 0.1$. The reconnection rate is the ratio of plasma inflow velocity and the local Alfvén velocity in the inflow region. Such rates are typically observed in numerical simulations of fast collisionless reconnection [10]. A reconnection rate of 0.1 corresponds to an inflow velocity of $\sim 25 \mathrm{~km} / \mathrm{s}$ or a tangential electric field in the magnetopause reference frame, $E_{\text {tang }}$, of $\sim 1 \mathrm{mV} / \mathrm{m}$. The observed $E_{\text {tang }}$ is varying but on average $\sim-1 \mathrm{mV} / \mathrm{m}$ [panel 2(e)]. This magnitude and sign of $E_{\text {tang }}$ are consistent with magnetic field observations, and simulations, of fast reconnection. Panel 2(f) shows that in the center of the current sheet (plasma outflow region) the density increases significantly by $\sim 50 \%$, and there is a similarly large density dip when entering the current sheet, consistent with the simulations. Reference [4] attributed a density dip observed by Wind in the magnetotail to the density dip predicted by simulations near the separatrices, but the exact location and the stability of this dip could not be determined. Here, we can tell that it is a spatial structure and it is located at separatrices. There is no significant density gradient across the magnetopause, thus, reconnection is almost symmetric. The absence of a significant density gradient across the magnetopause during this event can be explained by reconnection occurring between the so called plasma mantle (solar wind plasma that has entered the magnetosphere at some very distant reconnection site) and the magnetosheath. The slight differences between the separatrices [e.g., panels 2(d) and 2(f)] may be due to different plasma flow velocities observed before entering and after exiting the current sheet; the velocity shear is $\sim 150 \mathrm{~km} / \mathrm{s}$.

Simulations predict that a quadrupolar out-of-plane magnetic field structure in the diffusion region is caused by current loops that are mainly perpendicular to the ambient magnetic field in the center of the current sheet and mainly parallel near the separatrices, directed away from the $X$ line. The evidence for Hall currents has been reported in the form of the detection of field-aligned electrons flowing toward the $X$ line [4]. However, these observations do not allow the quantitative determination of the size of the current since the relative ion-electron motion is not known from these studies. Here we can do it by using four spacecraft to define the appropriate coordinate system and then using single spacecraft magnetic field perturbations to obtain the current. Panel 3(a) shows $B_{M}$ and Panel 3(b) shows the parallel current. As predicted by simulations, strong parallel currents occur along the outer edge of the bipolar $B_{M}$ structure. Panel 3(c) shows the electric field power integrated over a broad frequency range that includes the plasma frequency. The regions of strong emissions are clearly correlated with
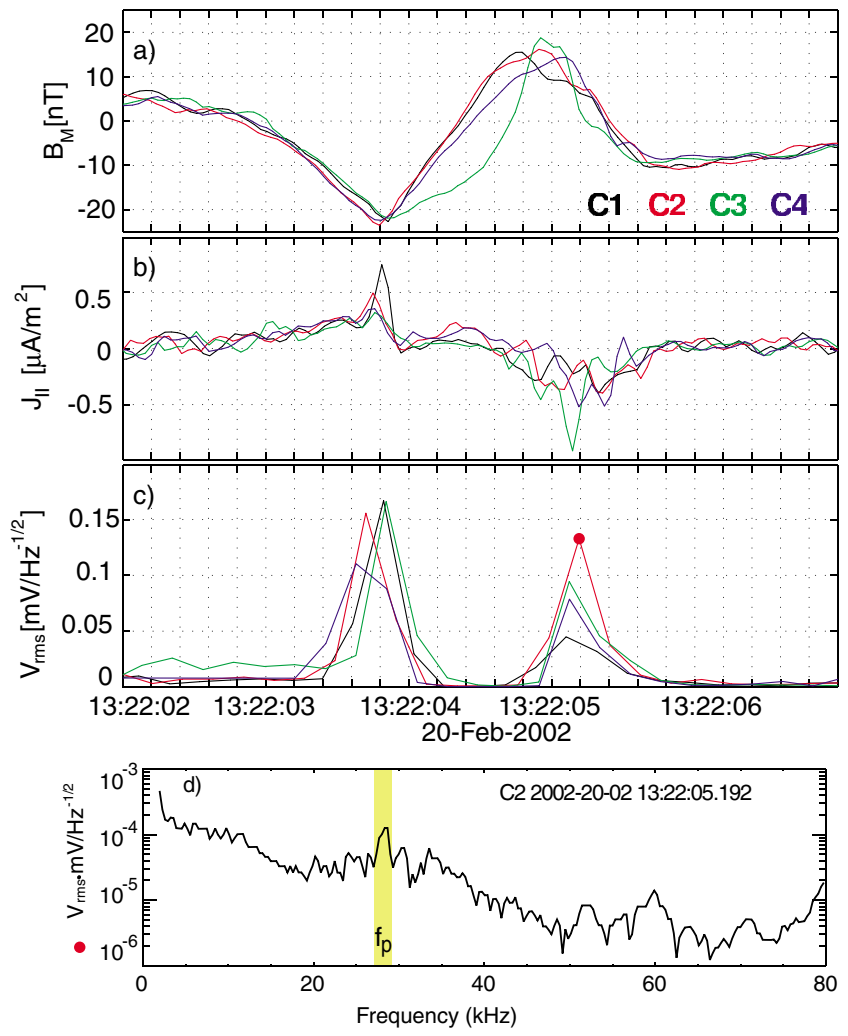

FIG. 3 (color online). Observations of field-aligned currents and Langmuir/upper hybrid waves at the separatrices. The same time interval as in Fig. 2, for Clusters 2, 3, and 4 the time series have been shifted $-0.09,-0.3$, and +0.55 s so that the observations are in the magnetopause frame. (a) Out-ofplane magnetic field component. (b) Current parallel to the magnetic field; the direction is away from the $X$ line. (c) Total spectral density in the frequency range $2-80 \mathrm{kHz}$ (integration from several times the electron gyrofrequency up to several times the electron plasma frequency). (d) Wave spectrum; strong emissions are seen observed near the plasma frequency, $f_{p} \sim 28 \mathrm{kHz}$. 
regions of strong parallel currents. Panel 3(d) shows the spectra of waves from $\mathrm{C} 2$ in one of the strong emission regions. There is a spectral peak near the plasma frequency, thus the waves are probably Langmuir or upper hybrid waves [11]. For the first time we can directly relate high-frequency waves in the vicinity of a reconnection region with the parallel currents of the separatrices.

In summary, using multispacecraft observations we can establish the spatial structure and stability of the current layer in the diffusion region of magnetic reconnection at the magnetopause. This allows us to resolve the spatial and temporal ambiguity that has always been present in the single spacecraft measurements. The diffusion region is stable on the ion time scale and its structure is consistent with theoretical predictions of fast reconnection that is governed by Hall physics. The reconnection rate is fast, $\sim 0.1$, and the bipolar out-ofplane and normal magnetic fields are quantitatively consistent with theoretical expectations. Such out-of-plane fields have been suggested as an indication of fast reconnection also by single spacecraft measurements with all the involved ambiguities. Here, in addition, we show that the large electric fields at the separatrices are balanced by the Hall term, $E_{n} \sim \mathbf{j} \times \mathbf{B} / n e \cdot \hat{\mathbf{n}}$, thus establishing the role of the Hall term in forming the structure and dynamics of the diffusion region. Other features are also consistent with predictions from numerical simulations: a bifurcated current sheet, density dips at the separatrices and a density increase in the center of current sheet. Multispacecraft measurements allow us to unambiguously resolve parallel currents along the separatrices and show that they are correlated with high-frequency Langmuir/upper hybrid waves. These waves can be involved in thermalization of electrons, formation of anomalous resistivity, and can be used as a diagnostics tool of reconnection sites. The good agreement between simulations and observations is a strong motivation to go further and study such important unanswered questions as whether reconnection at the magnetopause is always fast, whether the microphysics of the diffusion region affects the global processes, and what are the conditions for switch-on and switch-off of the reconnection process.

We thank A. I. Eriksson for valuable discussions and R. Isaksson for the event identification. We thank P.-A. Lindqvist for data support. AV research is supported by Swedish Research Council.

[1] G. Paschmann, I. Papamastorakis, N. Sckopke, G. Haerendel, B. U. O. Sonnerup, S. J. Bame, J. R. Asbridge, J.T. Gosling, C.T. Russel, and R. C. Elphic, Nature (London) 282, 243 (1979).

[2] F. S. Mozer, S. D. Bale, and T. D. Phan, Phys. Rev. Lett. 89, 015002 (2002).

[3] T. Nagai, I. Shinohara, M. Fujimoto, M. Hoshino, Y. Saito, S. Machida, and T. Mukai, J. Geophys. Res. 106, 25929 (2001).

[4] M. Øieroset, T. D. Phan, M. Fujimoto, R. P. Lin, and R. P. Lepping, Nature (London) 412, 414 (2001).

[5] C. P. Escoubet, C. T. Russell, and R. Schmidt, The Cluster and PHOENIX Missions (Dordrecht, Kluwer, 1997), edited by C. P. Escoubet, C. T. Russell, and R. Schmidt.

[6] B. N. Rogers, R. E. Denton, J. F. Drake, and M. A. Shay, Phys. Rev. Lett. 87, 195004 (2001).

[7] M. E. Mandt, R. E. Denton, and J. F. Drake, Geophys. Res. Lett. 21, 73 (1994).

[8] A. Runov et al., Geophys. Res. Lett. 30, 1579 (2003).

[9] M. André, A. Vaivads, S. C. Buchert, A. N. Fazakerley, and A. Lahiff, Geophys. Res. Lett. 31, L03803 (2004).

[10] M. A. Shay, J. F. Drake, B. N. Rogers, and R. E. Denton, J. Geophys. Res. 106, 3759 (2001).

[11] W. M. Farrell, M. D. Desch, M. L. Kaiser, and K. Goetz, Geophys. Res. Lett. 29, 1580 (2002). 